\title{
THE CARINA PROJECT. VII. TOWARD THE BREAKING OF THE AGE-METALLICITY DEGENERACY OF RED GIANT BRANCH STARS USING THE $C_{\mathrm{U}, \mathrm{B}, \mathrm{I}}$ INDEX
}

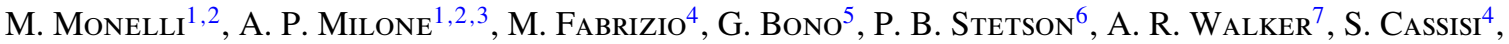

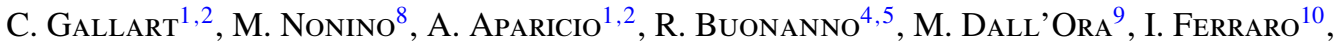 \\ G. IAnNiCOla ${ }^{10}$, L. PulOne $^{10}$, And F. Thévenin ${ }^{11}$ \\ ${ }^{1}$ Instituto de Astrofísica de Canarias, Calle Via Lactea s/n, E-38205 La Laguna, Tenerife, Spain; monelli@iac.es \\ ${ }^{2}$ Departamento de Astrofísica, Universidad de La Laguna, E-38200 La Laguna, Tenerife, Spain \\ ${ }^{3}$ Research School of Astronomy and Astrophysics, The Australian National University, Cotter Road, Weston, ACT 2611, Australia \\ ${ }^{4}$ Istituto Nazionale di Astrofisica-Osservatorio Astronomico Collurania, Via M. Maggini, I-64100 Teramo, Italy \\ ${ }^{5}$ Dipartimento di Fisica, Universitá di Roma Tor Vergata, Via della Ricerca Scientifica 1, I-00133 Rome, Italy \\ ${ }^{6}$ Dominion Astrophysical Observatory, NRC-Herzberg, 5071 West Saanich Road, Victoria, BC, V9E 2E7, Canada \\ ${ }^{7}$ Cerro Tololo Inter-American Observatory, National Optical Astronomy Observatory, Casilla 603, La Serena, Chile \\ ${ }^{8}$ Istituto Nazionale di Astrofisica-Osservatorio Astronomico di Trieste, Via G. B. Tiepolo 11, I-40131 Trieste, Italy \\ ${ }^{9}$ INAF-Osservatorio Astronomico di Capodimonte, Salita Moiariello 16, I-80131 Napoli, Italy \\ ${ }^{10}$ Istituto Nazionale di Astrofisica-Osservatorio Astronomico di Roma, Via Frascati 33, Monte Porzio Catone, I-00044 Rome, Italy \\ ${ }^{11}$ Université de Nice Sophia-antipolis, CNRS, Observatoire de la Côte d'Azur, Laboratoire Lagrange, BP 4229, F-06304 Nice, France \\ Received 2013 June 8; accepted 2014 September 25; published 2014 November 12
}

\begin{abstract}
We present an analysis of photometric and spectroscopic data of the Carina dSph galaxy, testing a new approach similar to that used to disentangle multiple populations in Galactic globular clusters (GCs). We show that a proper color combination is able to separate a significant fraction of the red giant branch (RGB) of the two main Carina populations (the old one, $\sim 12 \mathrm{Gyr}$, and the intermediate-age one, $4-8 \mathrm{Gyr}$ ). In particular, the $c_{\mathrm{U}, \mathrm{B}, \mathrm{I}}=(U-B)-(B-I)$ pseudo-color allows us to follow the RGB of both populations along a relevant portion of the RGB. We find that the oldest stars have a more negative $c_{\mathrm{U}, \mathrm{B}, \mathrm{I}}$ pseudo-color than intermediateage ones. We correlate the pseudo-color of RGB stars with their chemical properties, finding a significant trend between the iron content and the $c_{\mathrm{U}, \mathrm{B}, \mathrm{I}}$. Stars belonging to the old population are systematically more metal-poor $([\mathrm{Fe} / \mathrm{H}]=-2.32 \pm 0.08 \mathrm{dex})$ than the intermediate-age ones $([\mathrm{Fe} / \mathrm{H}]=-1.82 \pm 0.03 \mathrm{dex})$. This gives solid evidence of the chemical evolution history of this galaxy, and we have a new diagnostic that can allow us to break the age-metallicity degeneracy of H-burning advanced evolutionary phases. We compared the distribution of stars in the $c_{\mathrm{U}, \mathrm{B}, \mathrm{I}}$ plane with theoretical isochrones, finding that no satisfactory agreement can be reached with models developed in a theoretical framework based on standard heavy element distributions. Finally, we discuss possible systematic differences when compared with multiple populations in GCs.
\end{abstract}

Key words: galaxies: abundances - galaxies: individual (Carina dSph) - Local Group - techniques: photometric techniques: spectroscopic

Online-only material: color figures

\section{INTRODUCTION}

The classical question of how galaxies in general formed and evolved is one that can be illuminated by the study of galaxies in the Local Group (LG), particularly the numerous dwarf galaxies that are thought in some respects (mass, low metals) to be similar to the building blocks that have and are still being accreted by more massive galaxies such as our own Galaxy and M31. The characterization of the resolved stellar populations in terms of age and chemical content of dwarf galaxies in the LG has been the target of large observational efforts in recent decades (e.g., Gallart et al. 1996; Gallagher et al. 1998; Held et al. 1999; Aparicio et al. 2001; Bellazzini et al. 2001; Carrera et al. 2002; Dolphin et al. 2001; Skillman et al. 2003; Momany et al. 2005; McConnachie \& Irwin 2006; Cole et al. 2007; Rizzi et al. 2007; Walker et al. 2009; Martin et al. 2009; Monelli et al. 2010a, 2010b; Sanna et al. 2010; Hidalgo et al. 2011; Battaglia et al. 2012; Dall'Ora et al. 2012; de Boer et al. 2012; Okamoto et al. 2012).

Until recently, dwarf galaxies were thought to be fundamentally different from globular clusters (GCs). However, the recognition that many, if not all, GCs contain multiple populations (Kraft et al. 1992; Cannon et al. 1998; Grundahl et al. 1999;
Bedin et al. 2004; Marino et al. 2008; Milone et al. 2008; Yong \& Grundahl 2008; Lee et al. 2009; Piotto et al. 2012; Monelli et al. 2013) and that some of the most massive clusters may be the remnants of dissipated dwarf galaxies (Bekki et al. 2007) has blurred the differences somewhat. When considering the buildup of the halo of our own Galaxy, its chemical evolution is likely to be intimately intertwined with that of the GCs and accreting dwarf galaxies. The first high-resolution spectra for a handful of stars in nearby dSphs (Shetrone et al. 2001, 2003; Tolstoy et al. 2003) disclosed a systematic difference in the chemical enrichment law compared to the halo stars. This implies that the building blocks of the halo cannot resemble the present-day Galaxy satellites, unless the merging events occurred very early on, before the internal chemical evolution driven by supernova enrichment took place. On the other hand, it has recently been suggested that GCs might be responsible for a significant fraction of the halo stars observed today (D'Ercole et al. 2008). In this scenario, the GCs formed two or more generations of stars, on a short timescale and of the order of few hundred megayears, and the first supernovae would be responsible for significant mass loss from the clusters, causing the evaporation of a large fraction of the first generation stars; these would build up part of the Galaxy halo. Hence, a detailed knowledge 
of the chemical evolution of nearby dwarfs and a comparison with GCs can give important insight on the progenitors of our own Galaxy.

Ideally, we would like to collect high-resolution spectroscopy for large samples of stars to faint magnitudes, in conjunction with accurate photometry. However, for LG galaxies, the former is technically at present beyond our means and only typically the brightest stars in the closer galaxies have such data. Deep photometry alone suffers from the longstanding problem of the photometric color-magnitude diagram (CMD) having an age-metallicity degeneracy that affects the advanced evolutionary phases such as the red giant branch (RGB), where sequences of different age-metallicity combinations occupy the same region of the CMD and therefore cannot be easily disentangled.

Interestingly, recent results revealed that the different stellar populations in old GCs can be easily isolated along the whole $\mathrm{CMD}$, from the main sequence (MS), up to the subgiant branch (SGB), RGB, and even the horizontal branch, by using an appropriate combination of broadband filters (Marino et al. 2008; Sbordone et al. 2011; Milone et al. 2012c). Monelli et al. $(2013)^{12}$ showed that the $c_{\mathrm{U}, \mathrm{B}, \mathrm{I}}=(U-B)-(B-I)$ index is a powerful tool to identify multiple stellar sequences in the RGB of old GCs, and that the $c_{\mathrm{U}, \mathrm{B}, \mathrm{I}}$ pseudo-color of RGB stars correlates with the chemical abundances of light elements.

In this paper, we test the same approach successfully used for GCs for the first time in a dwarf galaxy. Since the established nomenclature can be somehow confusing, it has to be clearly stated that when we refer to the stellar populations in a dwarf galaxy, in particular the dSph satellites of the Milky Way, these have substantially different properties from multiple populations in GCs. First, the typical main star formation event in a dwarf galaxy occurred on timescales of the order of a few gigayears, significantly longer than the age difference between two generations of stars in a GC (a few hundred megayears at most). Moreover, the chemical evolution is different. In particular, the large spread in Fe observed in dwarf galaxies has no counterpart in a typical GC. In these systems, the internal chemical enrichment affected only the light elements involved in the high-temperature $\mathrm{H}$-burning processes, while typically no spread in Fe is found.

In this paper, we focus on the properties of the stellar population in the Carina $\mathrm{dSph}$ for which an extensive database of both photometric (Bono et al. 2010; Battaglia et al. 2012) and spectroscopic (Koch et al. 2006; Helmi et al. 2006; Fabrizio et al. 2011, 2012; Venn et al. 2012; Lemasle et al. 2012) data is available. Nevertheless, we still lack a definitive understanding of its bursty star formation history (Smecker-Hane et al. 1996; Monelli et al. 2003; Stetson et al. 2011), unique in the LG, and of the chemical enrichment history of its different stellar populations. In fact, an accurate analysis of high-quality photometry revealed that Carina experienced two main events of star formation, separated by a quiescent phase lasting a few gigayears, which produced two well-separated SGBs. One corresponds to an old population ( $>10 \mathrm{Gyr}$ ) containing approximately onethird of the total number of stars, while the second belongs to an intermediate-age population made of $\sim 4-8$ Gyr old stars (Smecker-Hane et al. 1996; Monelli et al. 2003). The two SGBs merge in a narrow RGB, making it difficult to associate the individual iron abundances with the discrete SGB and MS (Koch et al. 2006; Fabrizio et al. 2012; Venn et al. 2012) observed in the CMD. This represents a major challenge to understanding the

12 http://www.iac.es/project/sumo complex star formation history and the chemical evolution of the Carina dSph.

The paper is organized as follows. In Section 2, we present the $c_{\mathrm{U}, \mathrm{B}, \mathrm{I}}$ diagram for Carina, and the correlation between the $c_{\mathrm{U}, \mathrm{B}, \mathrm{I}}$ pseudo-color and the Fe content of RGB stars. In Section 3, we discuss the properties of Carina's stars with theoretical isochrones and GCs. The conclusions in Section 4 close the paper.

\section{DATA ANALYSIS}

\subsection{Photometric Data: Splitting the Carina RGB}

The photometric data are already presented in Bono et al. (2010). In particular, we focus here on the central region only, where $U, B, V$, and $I$ data overlap over an area of $\approx 40^{\prime} \times 40^{\prime}$. The left panel of Figure 1 shows the $(V, B-I)$ CMD of 20,240 stars selected with the following criteria: (1) they are measured in the four bands, and (2) they are bona fide Carina stars, with the removal of most of the possible Galactic field stars and foreground galaxies following the procedure explained in Bono et al. (2010), and based on the position of the sources in the $(U-V),(B-I)$ color-color plane. The plot demonstrates a nicely cleaned CMD showing well-defined evolutionary sequences, in particular the two well-separated turnoffs and SGBs representing the old and intermediate-age populations, merging into the thin RGB. The few remaining field stars populate an almost vertical sequence at color $1.2<(B-I)<1.6$ mag (cyan symbols for the brightest ones). These stars are virtually impossible to clean, even using a color-color plane, because their position is almost indistinguishable from the Carina turnoff (TO) stars. Note that most of the stars bluer than the field sequence and brighter than $V=20$ mag are Anomalous Cepheids of Carina (Coppola et al. 2013). The orange crosses mark the faint part of the asymptotic giant branch (AGB) star sequence.

The right panel of the same figure shows the $\left(V, c_{\mathrm{U}, \mathrm{B}, \mathrm{I}}\right)$ pseudo-CMD of Carina. As discussed in detail by Monelli et al. (2013), the features in the $\left(V, c_{\mathrm{U}, \mathrm{B}, \mathrm{I}}\right)$ diagram are reversed compared to a standard CMD, at least in the metallicity range covered by the Carina stars. The $c_{\mathrm{U}, \mathrm{B}, \mathrm{I}}$ of MS stars gets larger (i.e., less negative) as the stars get brighter, and after the turnoff the stars evolve to lower index values along the two SGBs. In this plane, the two RGBs do not totally merge in a single narrow sequence, as happens in standard optical CMDs. Although some degree of overlap is present for $-1.70<c_{\mathrm{U}, \mathrm{B}, \mathrm{I}}<-1.6 \mathrm{mag}$, it is possible to follow the two RGB sequences that remain welldefined and run almost vertically and parallel from the SGB to the tip of the RGB. In particular, the RGB stars with more negative $c_{\mathrm{U}, \mathrm{B}, \mathrm{I}}$ are a sequitur of the SGB of the old population, while the more densely populated, higher $c_{\mathrm{U}, \mathrm{B}, \mathrm{I}}$ side of the RGB is the sequitur of the intermediate-age population. Only at the brightest magnitudes ( $V \lesssim 19 \mathrm{mag}$ ) do the two sequences diverge slightly. We stress that the photometric error is of the order of a few hundredths of magnitude along the whole RGB, and therefore cannot account for the observed spread in the $c_{\mathrm{U}, \mathrm{B}, \mathrm{I}}$ index. Note that the contamination due to field stars is negligible across the RGB, while in this plane the majority of AGB stars overlie the RGB sequences. For this reason, the latter have been removed from the following analysis.

To better characterize these two RGB sequences, we followed the procedure outlined in Figure 2. Bona fide RGB stars are shown as black open circles (left and central panels), while the red line, drawn by eye, is intended to represent the right edge of their distribution. Our selection includes only stars in the 


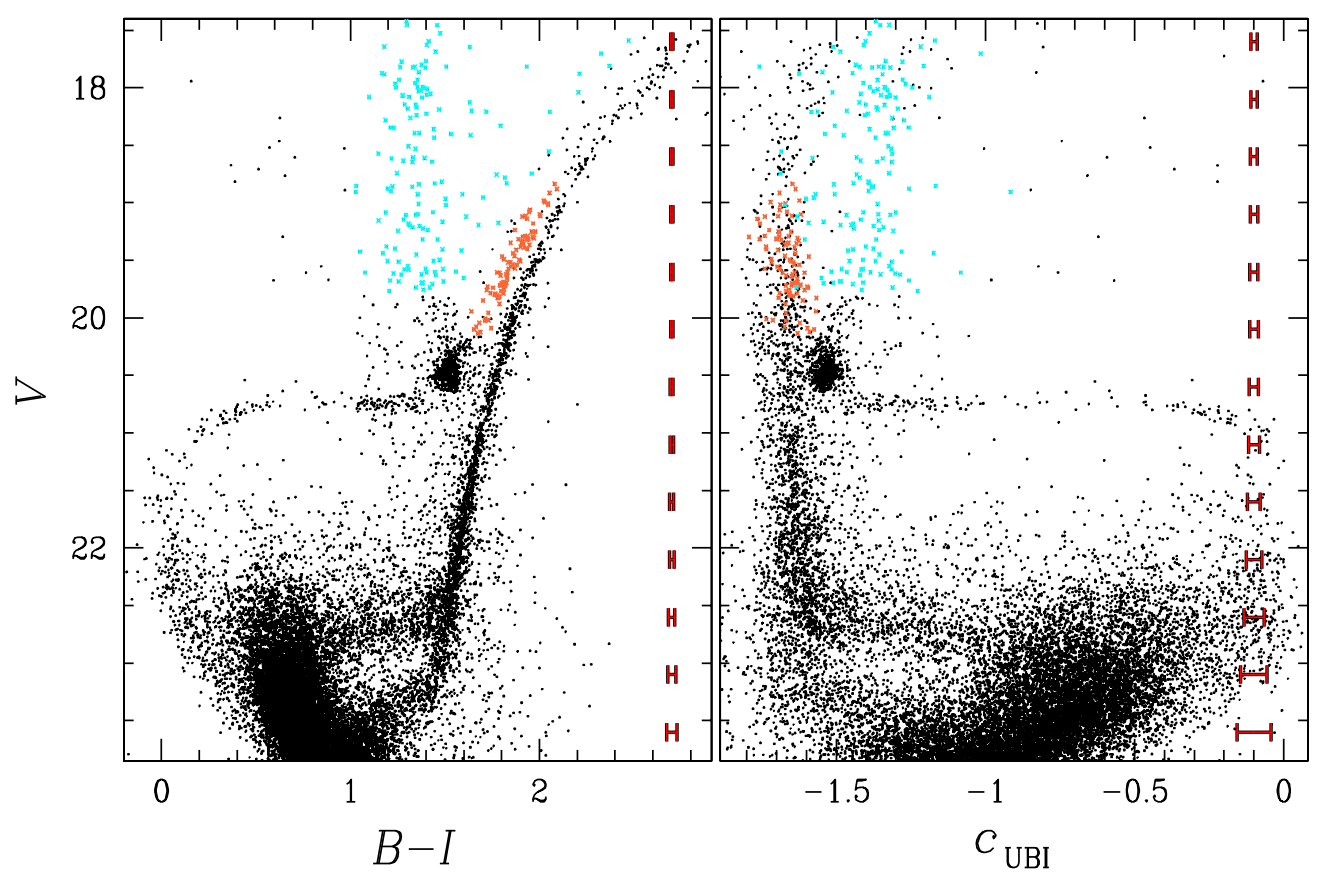

Figure 1. Left: $(V, B-I)$ CMD of Carina stars, based on the photometry presented in Bono et al. (2010). Bright field stars are highlighted in cyan, while orange symbols show the Carina AGB stars. Right: $\left(V, c_{\mathrm{U}, \mathrm{B}, \mathrm{I}}\right)$ plane, where the RGBs of the old and intermediate-age populations largely split.

(A color version of this figure is available in the online journal.)
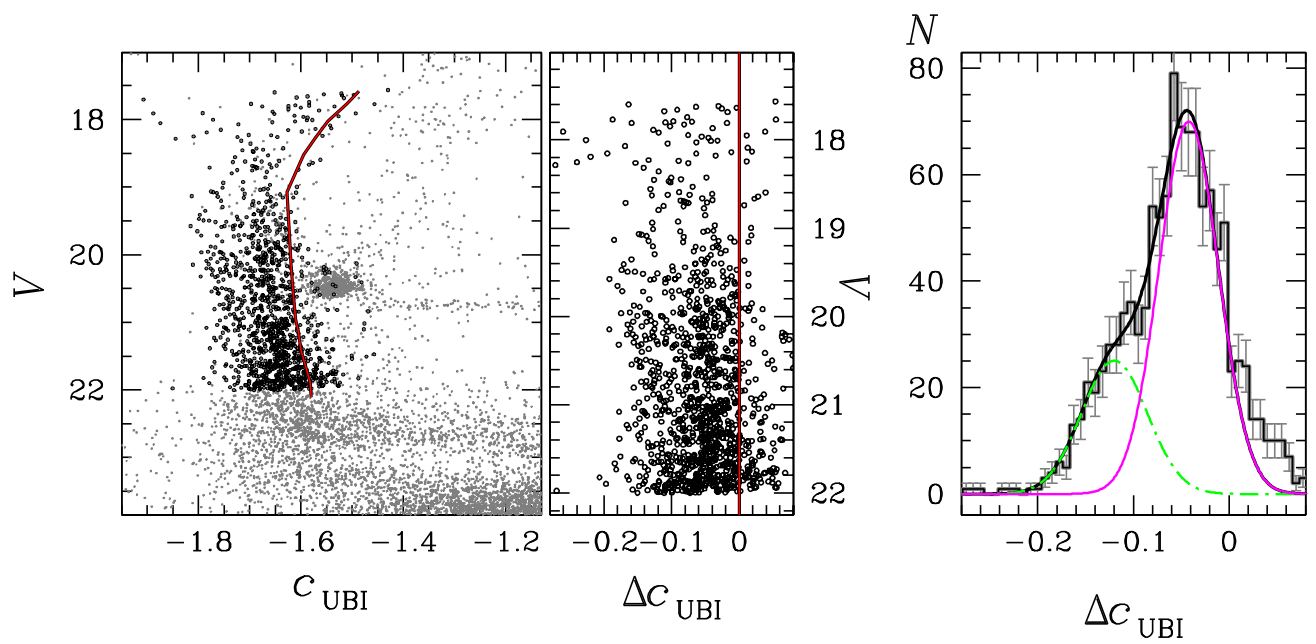

Figure 2. Left: the sample of RGB stars adopted to identify substructures in the RGB of Carina is shown as black open circles (1134 stars). The red line shows our adopted right envelope of the sequence. Center: the Carina RGBs, rectified to the red line. For each star, we display the $\Delta c_{\mathrm{U}, \mathrm{B}, \mathrm{I}}$ index, the color difference with respect to the reference red line. Right: histogram of $\Delta c_{\mathrm{U}, \mathrm{B}, \mathrm{I}}$, with Gaussian kernels superimposed. The overall distribution can be well represented by two Gaussian components.

(A color version of this figure is available in the online journal.)

magnitude range $17.5<V<22$ mag. Nevertheless, we verified that the following analysis is minimally affected, within $1 \sigma$, by both the exact location of the envelope and the limit magnitude. The RGB was rectified with respect to the red line, which is the origin of the abscissas in the central panel. This shows, for each star, the distance in color $\Delta c_{\mathrm{U}, \mathrm{B}, \mathrm{I}}$ from the red line. Finally, the right panel presents the distribution of the $\Delta c_{\mathrm{U}, \mathrm{B}, \mathrm{I}}$ (gray histogram). The shape of the global distribution is best fitted by the sum (black line) of the two superimposed Gaussian curves (green and magenta lines), whose peaks are separated by $\sim 0.08$ mag. This indicates that a simple photometric index such as the $c_{\mathrm{U}, \mathrm{B}, \mathrm{I}}$ is able to largely separate, for the first time, the two main components along the RGB of Carina. In particular, we conclude that stars with $c_{\mathrm{U}, \mathrm{B}, \mathrm{I}}<-1.70$ mag belong to the old population, while stars with less negative index values are predominantly intermediate-aged.

We verified this finding by considering only kinematically selected stars, adopting the sample of RGB stars from Fabrizio et al. (2011). This is shown in Figure 3, which is a replica of Figure 2 with 413 RGB stars with a radial velocity consistent with that of Carina superimposed. By adopting the selection from Fabrizio et al. (2011) and retaining stars with radial velocities within $4 \sigma$ from the mean value, in the range +180 to $+260 \mathrm{~km} \mathrm{~s}^{-1}$, the occurrence of a double peak in the pseudocolor distribution due to the two main Carina populations is confirmed. 

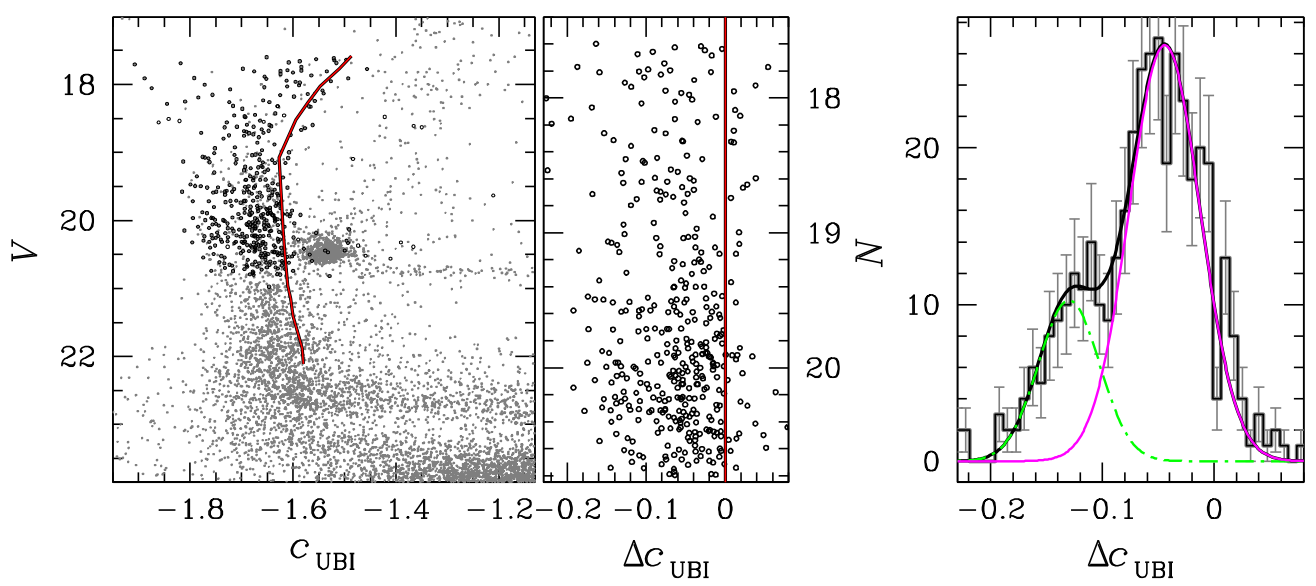

Figure 3. Same as Figure 2, but in this case the open circles in the left panel mark 413 RGB stars with radial velocities consistent with that of Carina, selected from the sample of Fabrizio et al. (2011). In the right panel, the presence of a double peak in the $\Delta c_{\mathrm{U}, \mathrm{B}, \mathrm{I}}$ distribution is confirmed.

(A color version of this figure is available in the online journal.)

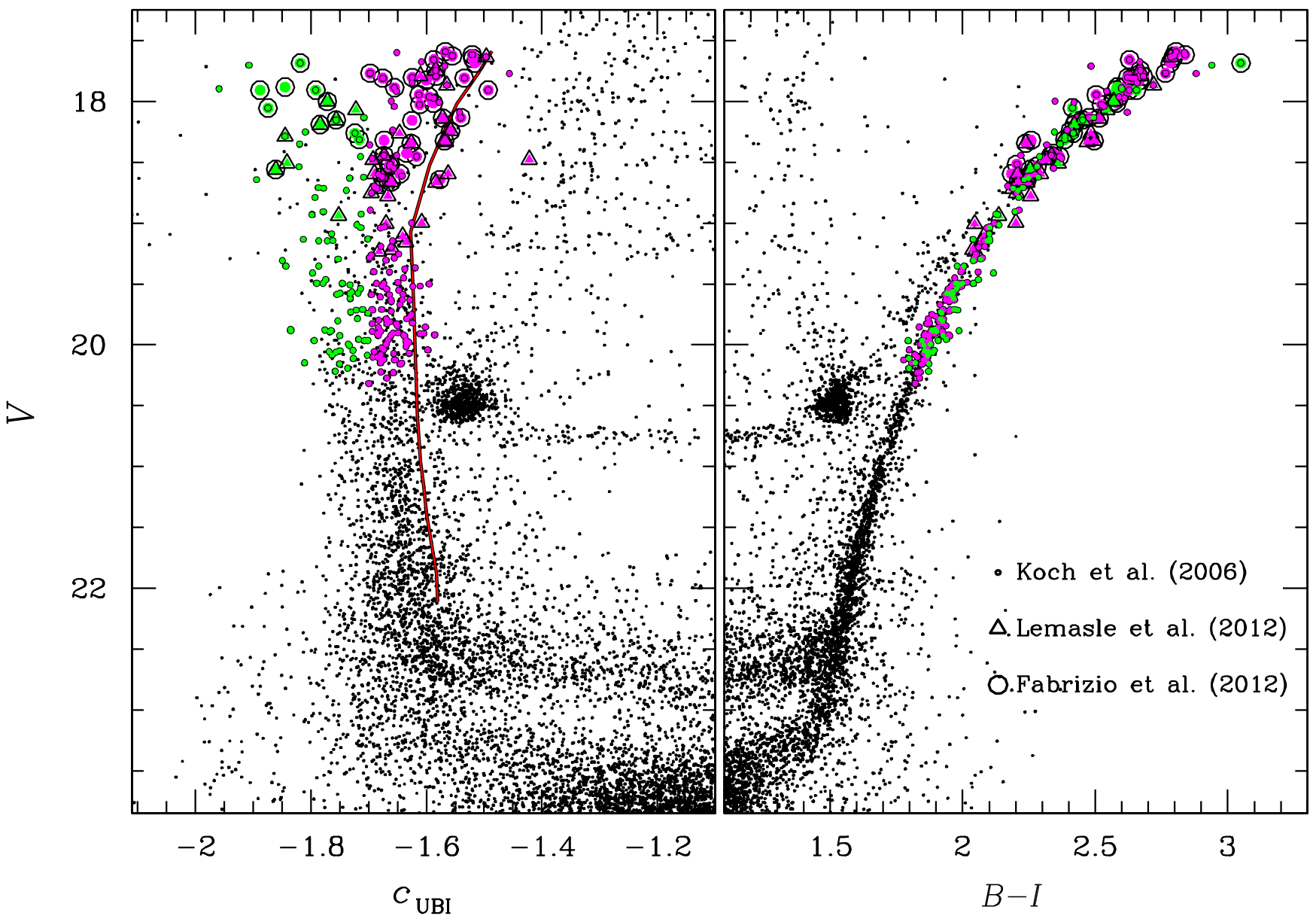

Figure 4. Left: $\left(V, c_{\mathrm{U}, \mathrm{B}, \mathrm{I}}\right)$ pseudo-CMD with the stars for which spectroscopic measurements are available superimposed. Large symbols show stars with available Fe abundances from high-resolution spectra (dots: Fabrizio et al. 2012; triangles: Lemasle et al. 2012). Small colored dots mark the 212 stars selected from Koch et al. (2006), for which the Fe abundance was derived from $\mathrm{CaT}$ measurements. Green and magenta symbols indicate stars with $c_{\mathrm{U}, \mathrm{B}, \mathrm{I}}<-1.70 \mathrm{mag}$ and $c_{\mathrm{U}, \mathrm{B}, \mathrm{I}}>-1.70 \mathrm{mag}$, respectively. Right: $(V, B-I)$ CMD showing the complete degeneracy of spectroscopic targets on the RGB, using the same color coding.

(A color version of this figure is available in the online journal.)

\subsection{Spectroscopic Data: Connecting Multiple Sequences and Metallicity}

In this section, we investigate whether a connection exists between the two sequences identified in the RGB and the chemical compositions of member stars. To do this, we use three independent samples of spectroscopic data available in the literature (see Figure 4). We adopt two samples of highresolution measurements of $\mathrm{Fe}$ lines from Fabrizio et al. (2012; $R \sim 40,000$, large circles) and Lemasle et al. (2012; $R \sim 22,000$, triangles). The two samples include 44 and 35 stars for a total of 68 red giants (11 in common). The entire sample of stars with high-resolution spectra has at least one photometric measurement in the 
Table 1

Mean Metallicities for the Different Spectroscopic Samples and Stellar Populations

\begin{tabular}{|c|c|c|c|c|c|c|}
\hline Spectroscopic Sample & $\langle[\mathrm{Fe} / \mathrm{H}]\rangle_{\text {old }}$ & $\sigma$ & $N_{\text {stars }}$ & $\langle[\mathrm{Fe} / \mathrm{H}]\rangle_{\text {int }}$ & $\sigma$ & $N_{\text {stars }}$ \\
\hline \multicolumn{7}{|l|}{ Cut at $c_{\mathrm{U}, \mathrm{B}, \mathrm{I}}=-1.70 \mathrm{mag}$} \\
\hline Fabrizio et al. (2012) & $-2.32 \pm 0.08$ & 0.25 & 10 & $-1.82 \pm 0.03$ & 0.20 & 34 \\
\hline Lemasle et al. (2012) & $-2.03 \pm 0.13$ & 0.35 & 8 & $-1.52 \pm 0.03$ & 0.18 & 27 \\
\hline Koch et al. (2006) $[V<19 \mathrm{mag}]$ & $-2.06 \pm 0.05$ & 0.24 & 24 & $-1.55 \pm 0.03$ & 0.24 & 47 \\
\hline Koch et al. (2006) $[V \geqslant 19 \mathrm{mag}]$ & $-1.96 \pm 0.05$ & 0.33 & 54 & $-1.56 \pm 0.04$ & 0.38 & 87 \\
\hline \multicolumn{7}{|l|}{ Cut at $c_{\mathrm{U}, \mathrm{B}, \mathrm{I}}=-1.67 \mathrm{mag}$} \\
\hline Fabrizio et al. (2012) & $-2.25 \pm 0.07$ & 0.27 & 14 & $-1.79 \pm 0.03$ & 0.16 & 30 \\
\hline Lemasle et al. (2012) & $-1.80 \pm 0.10$ & 0.37 & 15 & $-1.51 \pm 0.04$ & 0.18 & 20 \\
\hline Koch et al. (2006) $[V<19 \mathrm{mag}]$ & $-1.92 \pm 0.06$ & 0.34 & 36 & $-1.52 \pm 0.03$ & 0.19 & 35 \\
\hline Koch et al. (2006) $[V \geqslant 19 \mathrm{mag}]$ & $-1.85 \pm 0.04$ & 0.38 & 86 & $-1.50 \pm 0.05$ & 0.41 & 55 \\
\hline \multicolumn{7}{|l|}{ Cut at $c_{\mathrm{U}, \mathrm{B}, \mathrm{I}}=-1.73 \mathrm{mag}$} \\
\hline Fabrizio et al. (2012) & $-2.34 \pm 0.11$ & 0.28 & 8 & $-1.85 \pm 0.04$ & 0.21 & 36 \\
\hline Lemasle et al. (2012) & $-2.10 \pm 0.13$ & 0.31 & 7 & $-1.52 \pm 0.03$ & 0.17 & 28 \\
\hline Koch et al. (2006) $[V<19 \mathrm{mag}]$ & $-2.10 \pm 0.06$ & 0.25 & 18 & $-1.59 \pm 0.04$ & 0.26 & 53 \\
\hline Koch et al. (2006) $[V \geqslant 19 \mathrm{mag}]$ & $-1.93 \pm 0.05$ & 0.33 & 38 & $-1.63 \pm 0.04$ & 0.41 & 103 \\
\hline
\end{tabular}

four adopted filters, namely, $V$ and $U B I$ (see Figure 4). We also included red giant stars for which $\mathrm{Fe}$ abundances were determined by Koch et al. (2006) using medium-resolution $(R \sim 6000)$ spectra (small circles in Figure 4$)$ in the CaT region. Out of the initial catalog by Koch et al. (2006, see their Table 6), including 1197 stars, we selected 212 objects with an $[\mathrm{Fe} / \mathrm{H}]$ abundance and that obey the same photometric and radial velocity selection criteria adopted in Figures 1 and 2 . We note that in this analysis, we adopt the $[\mathrm{Fe} / \mathrm{H}]$ abundance from Koch et al. (2006) and the radial velocity from Fabrizio et al. (2011).

The analysis was performed by splitting all the spectroscopic samples according to their $c_{\mathrm{U}, \mathrm{B}, \mathrm{I}}$ index. In particular, green and magenta symbols account for stars with $c_{\mathrm{U}, \mathrm{B}, \mathrm{I}}<-1.70 \mathrm{mag}$ and $c_{\mathrm{U}, \mathrm{B}, \mathrm{I}}>-1.70 \mathrm{mag}$, respectively. For comparison, the right panel presents the $(V, B-I)$ classical CMD, where the two samples of stars selected according the $c_{\mathrm{U}, \mathrm{B}, \mathrm{I}}$ index are mixed. Figure 5 presents the metallicity distribution for the three samples. The sample from Koch et al. (2006) has been further split in two, separating the brightest (bottom left, $V<19$ mag, 71 stars) from the faintest (bottom right, $V \geqslant 19 \mathrm{mag}, 141$ stars) ones, to account for the change in the RGB morphology previously discussed (Figure 1). The plot discloses that the stars selected on the sequence with more negative $c_{\mathrm{U}, \mathrm{B}, \mathrm{I}}$ (green histogram), identified as the RGB of the old population, are on average more metal-poor than the stars on the other sequence (magenta), dominated by the intermediate-age population. The different independent samples provide a very good overall agreement, and in particular the sample of bright stars from Koch et al. (2006) notably agree with the high-resolution samples. In the case of the faint CaT stars, the overlap between the two histograms increases, possibly due to the increase in the observational errors. The mean metallicity of each sample is reported in Table 1 . We find that the mean metallicity of the old population is $\approx 0.5$ dex more metal-poor than the intermediateage one. ${ }^{13}$ Assuming the Fabrizio et al. (2012) data, which include stars with both Fe I and Fe II measurements, we find that the mean metallicities are $[\mathrm{Fe} / \mathrm{H}]=-2.32 \pm 0.08$ dex and $[\mathrm{Fe} / \mathrm{H}]=-1.82 \pm 0.04$ dex for the old and the intermediateage group, respectively. While previous investigations (see, e.g., Lemasle et al. 2012) have suggested a similar chemical evolution for Carina, we stress that our approach is independent

\footnotetext{
13 The difference in the absolute value of the Fe abundances between the
} different data sets has already been discussed in Fabrizio et al. (2012).
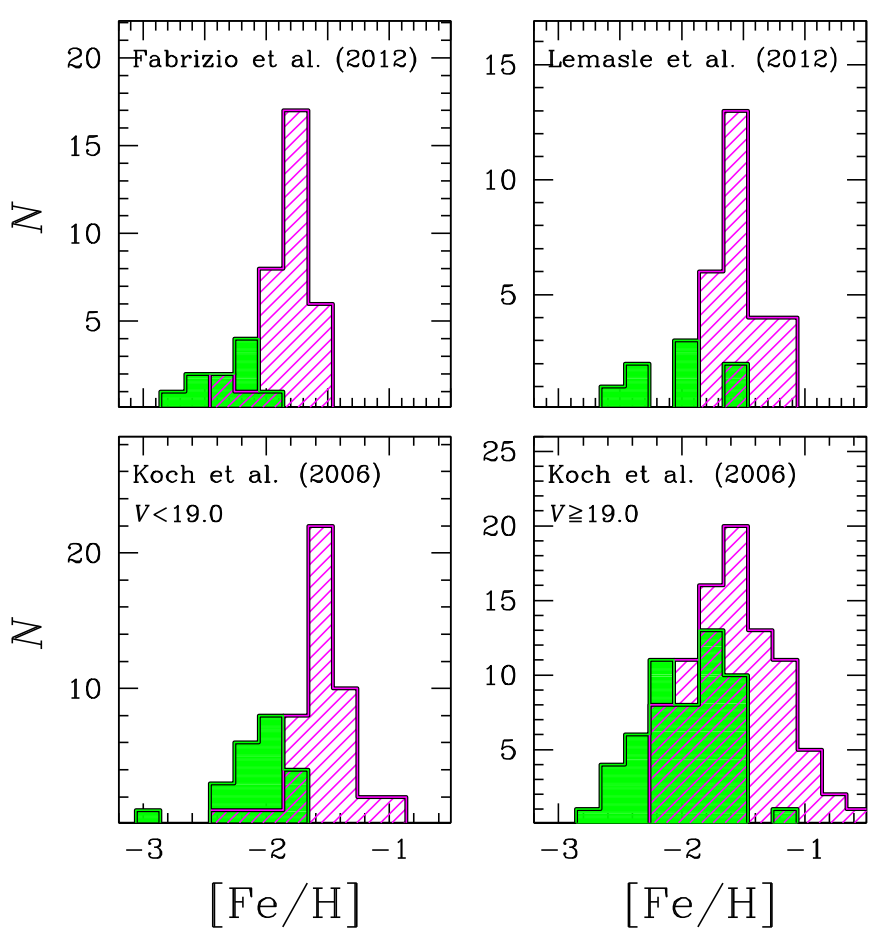

Figure 5. Metallicity distribution of the three spectroscopic samples. The top panels show the high-resolution samples: Fabrizio et al. (2012; 44 stars, left) and Lemasle et al. (2012; 35 stars, right). In the lower panels, the mediumresolution sample from Koch et al. (2006) was split in two subgroups: stars with $V<19$ mag (71, bottom left), and stars with $V \geqslant 19$ mag (141, bottom right). (A color version of this figure is available in the online journal.)

of isochrone fitting. Identification of RGB stars as members of the old or the intermediate-age population does not require previous knowledge of the metallicity.

The cut adopted to split the two quoted populations, $\left(c_{\mathrm{U}, \mathrm{B}, \mathrm{I}}=-1.73\right) \mathrm{mag}$, is, as noted by the anonymous referee, arbitrary. Mild changes in the adopted cut can move objects from the old sample into the intermediate-age one and vice versa. To constrain, on a quantitative basis, the impact of the adopted cut on the mean metallicity of the two samples, we performed the selection by adopting $c_{\mathrm{U}, \mathrm{B}, \mathrm{I}}=-1.67$ and $c_{\mathrm{U}, \mathrm{B}, \mathrm{I}}=-1.73 \mathrm{mag}$. The difference was fixed according to the photometric error of the $c_{\mathrm{U}, \mathrm{B}, \mathrm{I}}$ index at $V \sim 20.5\left(\sigma_{\mathrm{UBI}}=0.03 \mathrm{mag}\right)$. The mean metallicities listed in Table 1 further support that the criterion 


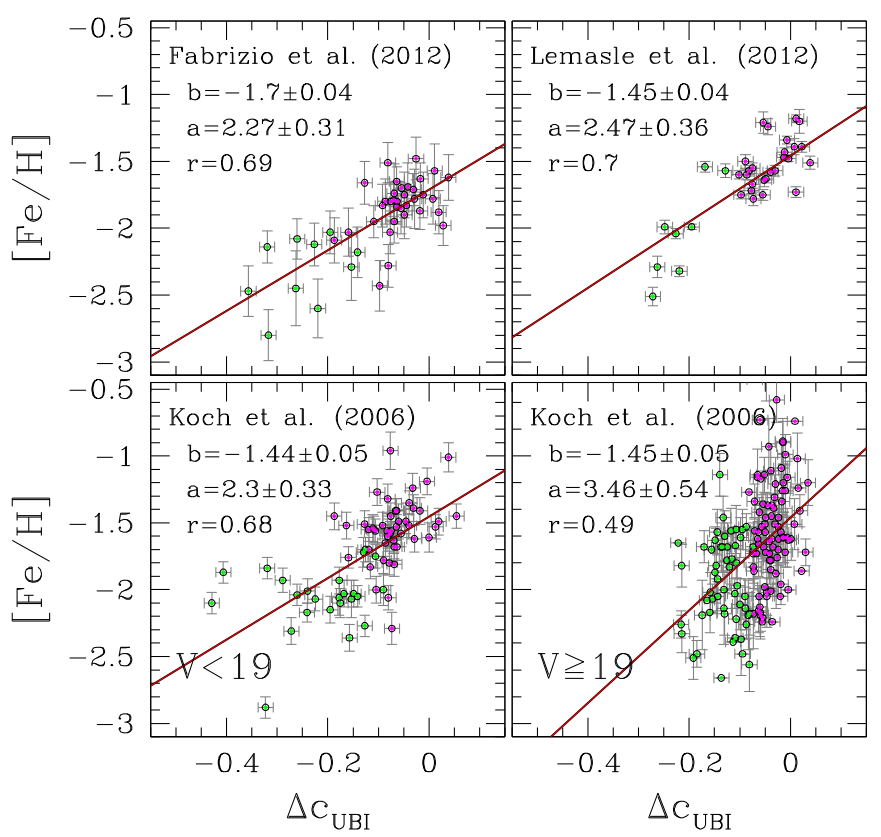

Figure 6. Top: correlation between the $c_{\mathrm{U}, \mathrm{B}, \mathrm{I}}$ color and the iron content of stars for the high-resolution samples. Bottom: the same is shown also for the Koch et al. (2006) sample. The slope of the relation changes according to the luminosity of the sample; see the text for details. Green and magenta symbols indicate stars with $c_{\mathrm{U}, \mathrm{B}, \mathrm{I}}<-1.70 \mathrm{mag}$ and $c_{\mathrm{U}, \mathrm{B}, \mathrm{I}}>-1.70 \mathrm{mag}$, respectively, as in Figures 4 and 5.

(A color version of this figure is available in the online journal.)

adopted to split the two samples minimally affects the current conclusions. In particular, the systematic difference between the mean metallicity of the old and the intermediate-age population is systematically, on average, larger than 0.5 dex. This result is an independent demonstration that the color spread observed in $c_{\mathrm{U}, \mathrm{B}, \mathrm{I}}$ is intrinsic. In fact, the $c_{\mathrm{U}, \mathrm{B}, \mathrm{I}}$ index and the metallicity used here have been measured in an independent way. If the RGB spread observed in $c_{\mathrm{U}, \mathrm{B}, \mathrm{I}}$ was entirely due to photometric errors (either statistical or systematic), a star with small (large) $c_{\mathrm{U}, \mathrm{B}, \mathrm{I}}$ would have the same probability of being either metalrich or metal-poor. Moreover, this result is supported by three independent sets of spectroscopic measurements.

Figure 6 presents the iron abundance as a function of the $\Delta c_{\mathrm{U}, \mathrm{B}, \mathrm{I}}$ parameter previously defined. The top panels show the trend for the two high-resolution samples, while the lower panels present the medium-resolution ones. The plots disclose a clear trend: the lower the $\Delta c_{\mathrm{U}, \mathrm{B}, \mathrm{I}}$, the lower the Fe abundance. We fit the data with a linear function in the form $f=a x+b$, reporting in each panel the values of both the zero point $(b)$ and the slope $(a)$, as well as the Spearman coefficient $(r)$, which suggests that the correlation is significant. The four samples provide a general good agreement, in particular concerning the bright portion of the RGB where both high and medium resolutions are present. The subsample of faint stars presents a steeper correlation. This can be easily explained, taking into account that the RGB morphology in the $c_{\mathrm{U}, \mathrm{B}, \mathrm{I}}$ plane changes as a function of magnitude. In the upper part, the two RGB sequences, where most of the high-resolution stars are, tend to diverge. This pushes the stars on the old RGB to lower $c_{\mathrm{U}, \mathrm{B}, \mathrm{I}}$, making the relation less steep. For magnitudes fainter than $V \sim 19 \mathrm{mag}$, the two RGB are closer, and this is reflected in a steeper relation between $\mathrm{Fe}$ and $\Delta c_{\mathrm{U}, \mathrm{B}, \mathrm{I}}$.

In Section 2.1, we mentioned that the cut at $c_{\mathrm{U}, \mathrm{B}, \mathrm{I}}=$ $-1.70 \mathrm{mag}$ to split the old and the intermediate-age is approximately equivalent to a cut at $\Delta c_{\mathrm{U}, \mathrm{B}, \mathrm{I}}=-0.1 \mathrm{mag}$.

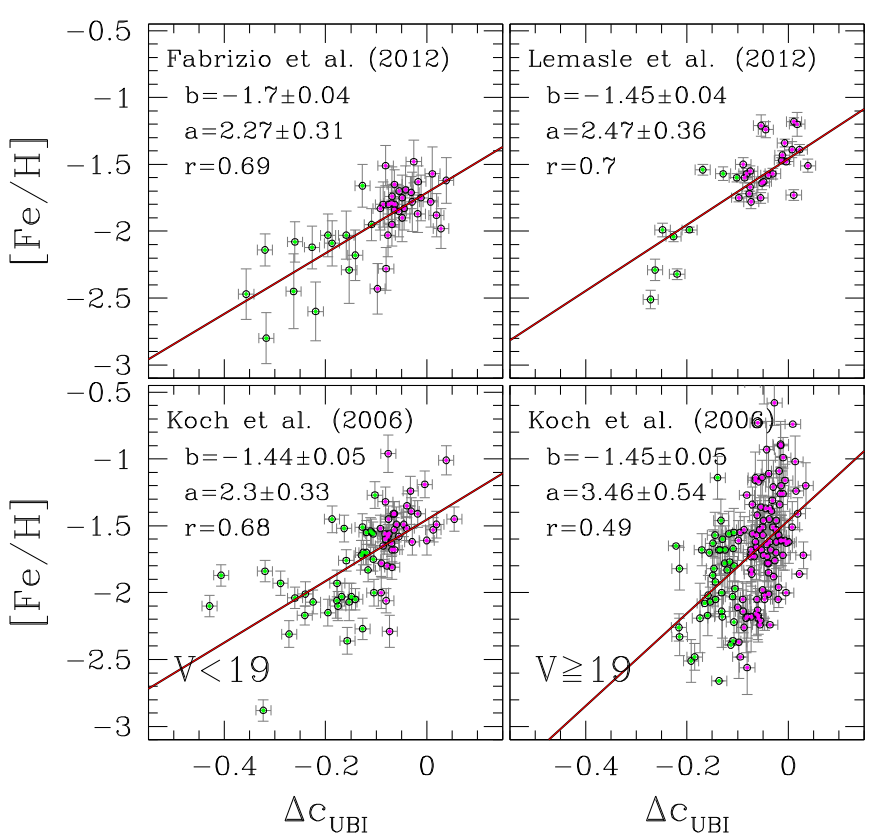

Figure 7. Same as Figure 6, but in this case green and magenta symbols show stars with $\Delta c_{\mathrm{U}, \mathrm{B}, \mathrm{I}}<-0.1 \mathrm{mag}$ and $\Delta c_{\mathrm{U}, \mathrm{B}, \mathrm{I}}>-0.1 \mathrm{mag}$, respectively. The plot demonstrates that the selection criterion does not affect the derived conclusions. (A color version of this figure is available in the online journal.)

The anonymous referee noted that the two quoted cuts are equivalent for the high-resolution sample by Lemasle et al. (2012; top right panel of Figure 6) and for the faint mediumresolution sample (bottom right panel of Figure 6). On the other hand, there are objects in the bright magnitude sample and in the high-resolution sample by Fabrizio et al. (2012; left panels of Figure 6) moving across the boundary of the $\Delta c_{\mathrm{U}, \mathrm{B}, \mathrm{I}}$ distribution. To take account of the role that the selection criterion has on the correlation between the $\Delta c_{\mathrm{U}, \mathrm{B}, \mathrm{I}}$ parameter and the iron abundance, we performed the same test, cutting the two populations at $\Delta c_{\mathrm{U}, \mathrm{B}, \mathrm{I}}=-0.1 \mathrm{mag}$. Data plotted in Figure 7 show that only a few stars near the edge are classified in a different population. Therefore, this means that we cannot reach a firm conclusion concerning the nature (old versus intermediate-age) of the objects that are located across the boundary $\left(c_{\mathrm{U}, \mathrm{B}, \mathrm{I}}=-1.70 \mathrm{mag}\right.$, $\Delta c_{\mathrm{U}, \mathrm{B}, \mathrm{I}}=-0.1 \mathrm{mag}$ ) adopted to split the two main populations. However, the above results further support evidence that the correlation is marginally dependent on the criterion adopted to split the two populations.

Finally, we stress that this analysis is not meant to find an absolute calibration of the $c_{\mathrm{U}, \mathrm{B}, \mathrm{I}}$ index as a function of metallicity. Nevertheless, the correlation supports the connection between the $c_{\mathrm{U}, \mathrm{B}, \mathrm{I}}$ index and the Fe content of the Carina RGB stars.

\section{DISCUSSION: STELLAR POPULATIONS AND CHEMICAL EVOLUTION IN CARINA}

\subsection{Comparison with Theoretical Models}

To assess whether the theoretical models are able to reproduce the observed color distribution of RGB stars, in the following we perform a comparison with theoretical models from the BaSTI $^{14}$ database (Pietrinferni et al. 2004). Figure 8 shows a comparison in the $\left(V, c_{\mathrm{U}, \mathrm{B}, \mathrm{I}}\right)$ plane with scaled-solar isochrones of three different metallicities $(Z=0.0001,0.0003,0.0006)$,

\footnotetext{
14 http://basti.oa-teramo.inaf.it/index.html
} 


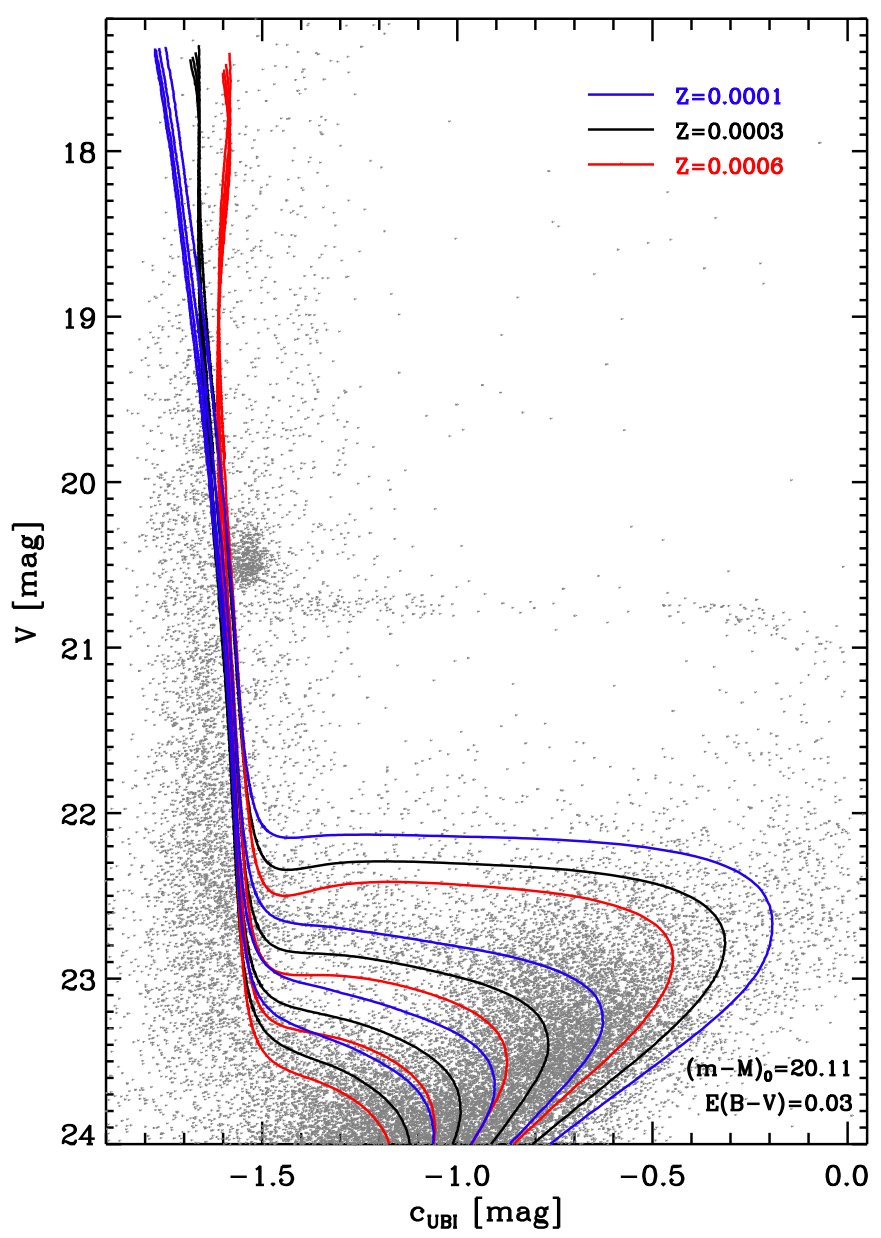

Figure 8. Comparison with theoretical isochrones from the BaSTI database in the $\left(M_{V}, c_{\mathrm{U}, \mathrm{B}, \mathrm{I}}\right)$ plane. For the three metallicities labeled, isochrones with ages between 4 and $13 \mathrm{Gyr}$, in steps of $3 \mathrm{Gyr}$, are plotted.

(A color version of this figure is available in the online journal.)

and ages ranging from 4 to $13 \mathrm{Gyr}$, in steps of $3 \mathrm{Gyr}$. The figure discloses that the color range covered by the selected isochrones $(\approx 0.03 \mathrm{mag}$ at $V=21 \mathrm{mag}$ ) is significantly smaller than the observed one in the $\left(V, c_{\mathrm{U}, \mathrm{B}, \mathrm{I}}\right)$ plane. Interestingly, in the upper part of the RGB ( $V \lesssim 19.5 \mathrm{mag}$ ), the effect of metallicity is to split the isochrones, in agreement with the observed increasing separation of the Carina RGB sequences, discussed in Figure 1. For fainter magnitudes, it is not possible to disentangle the isochrones of different metallicity, at least in the range typical of the Carina Fe content. The effect of age along the RGB is largely negligible in this plane for both the old and the intermediate-age population. Overall, we conclude that current models based on a standard heavy-element distribution do not account for the spread in $c_{\mathrm{U}, \mathrm{B}, \mathrm{I}}$ observed in the Carina RGBs.

These results do not substantially change when using $\alpha$ - or He-enhanced models (see also Cassisi et al. 2013a, 2013b). Current theoretical framework does not allow us to fully explain the behavior of the two sequences in the $c_{\mathrm{U}, \mathrm{B}, \mathrm{I}}$ plane by accounting for iron, age, $\alpha$ elements, and He differences alone. We remark that this cannot be due to a residual shortcoming in the adopted color- $T_{\text {eff }}$ transformations because we are using model predictions in a strictly differential way.

\subsection{Comparison with GCs}

Figure 9 shows how stars of the two sequences are distributed in different CMDs, namely $U-I, B-I$, and $V-I$ (top row, from

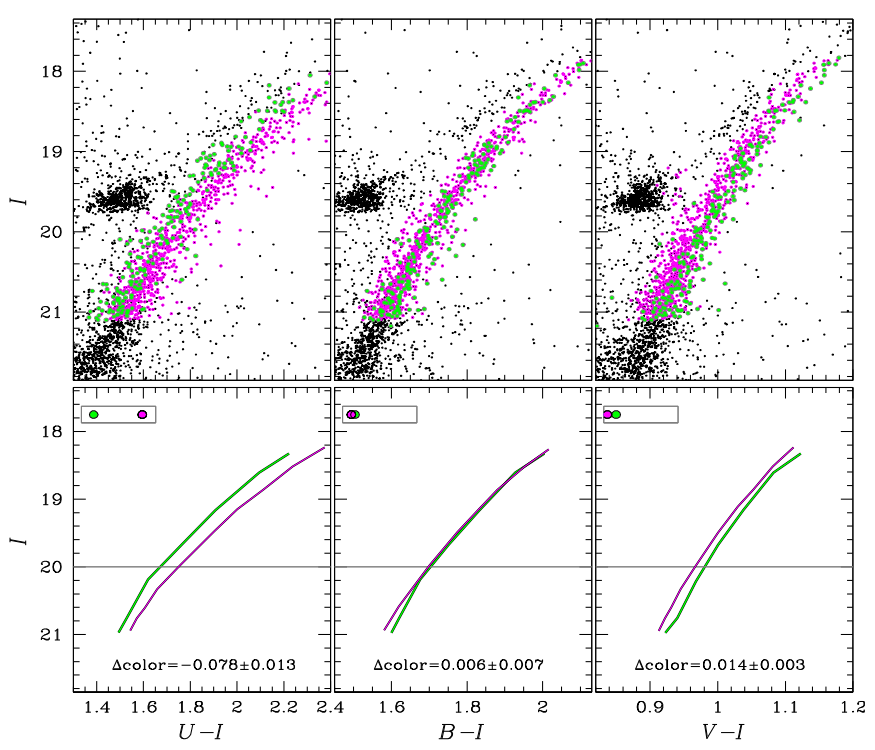

Figure 9. Top: $I$ vs. $U-I, B-I, V-I$ CMDs zoomed in the RGB region, with stars from the two RGBs selected in the $\Delta c_{\mathrm{U}, \mathrm{B}, \mathrm{I}}$ highlighted in green (old population) and magenta (intermediate-age). Bottom: ridge lines of the two populations. Note the change in the relative position for decreasing color baseline. The mean color difference with respect to the green line at magnitude $I=20 \mathrm{mag}$ is labeled and illustrated by the colored points in the top-left corner of each panel.

(A color version of this figure is available in the online journal.)

left to right). The two sequences appear overlapped and mixed, and therefore indistinguishable, in $B-I$, while they are slightly segregated in $V-I$ and substantially more so in $U-I$. However, the two sequences are swapped in two planes: in $U-I$ the green symbols (old stars) are concentrated on the blue edge of the RGB, while the magenta circles (intermediate-age population) are located closer to the red edge. The opposite occurs for the $V-I$ color. This is evident in the lower panels, where we show the fiducial lines of the two samples. To quantify the split, we calculate the color difference at a magnitude level of $I=20 \mathrm{mag}$ - that is, roughly two magnitudes below the tip of the RGB. As expected, we find that the separation is negligible in $B-I$, and only slightly larger in $V-I$, on the order of $0.01 \mathrm{mag}$, which is statistically significant given the typical photometric error at this magnitude level. The largest separation is found in the $U-I$ color, of the order of $-0.08 \mathrm{mag}$.

It is instructive to compare the behavior of the RGBs in Carina with similar observations of multiple RGBs in GCs. In several GCs, the RGB and the MS of first-population stars are redder than the second-population RGB and MS in $B-I$ and $V-I$ colors, and the color separation increases for increasing color baseline (that is, $\Delta(B-I)>\Delta(V-I)>0)$. However, the RGBs can merge together or even change their relative positions when using a near-UV filter $(\Delta(U-I)<0)$. On the other hand, if farultraviolet (FUV) filters are adopted, the separation between the two RGBs increases again and is significantly larger in FUV - I than in visual colors (see Milone et al. 2012a, 2012b).

This has been explained as being due to the detailed abundances of the various light elements (e.g., C, N, Na, O) and helium. In particular, the colors of the first-generation stars in GCs are well reproduced assuming primordial He abundance and an $\mathrm{O}$-rich/N-poor composition that is similar to that of halo-field stars of the same metallicity. On the other hand, the colors of the second-population stars agree with a composition in which $\mathrm{N}$ and $\mathrm{Na}$ are enhanced-along with increased $\mathrm{He}-$ while $\mathrm{C}$ and 
$\mathrm{O}$ are depleted. Sbordone et al. (2011) have demonstrated that such abundance variations mostly affect the $\mathrm{CN}, \mathrm{NH}$, and $\mathrm{CH}$ bands in the UV part of the spectrum, and this translates into different colors for the different populations when $U V$ filters are used. These effects can be well reproduced by stellar models if the appropriate chemical mixtures are adopted (Sbordone et al. 2011).

Results shown in Figure 9 demonstrate that the RGB behavior of stellar populations in the Carina dSph and in GCs differs significantly. Carina is indeed an intrinsically more complicated system than a typical GC, due to the bursty star formation history and the large age and metallicity spread present in each population. Multiple populations in a GC formed on a much shorter timescale, and, in most cases, the internal chemical enrichment affected only the light elements involved in the hightemperature H-burning processes, while typically no spread in iron due to supernova pollution is found.

Moving to a more speculative scenario, a possible explanation for the empirical evidence is that light-element abundances could play a non-negligible role in determining the behavior of the multiple RGBs in the Carina dSph, in analogy to what is observed in GCs. The $c_{\mathrm{U}, \mathrm{B}, \mathrm{I}}$ index is very sensitive to lightelement variations, which are measured in dwarf galaxies as well. However, it is not clear at present whether the chemical patterns are similar to that of GCs. In fact, the $\mathrm{Na}-\mathrm{O}$ and $\mathrm{C}-\mathrm{N}$ anti-correlations have not been observed for $\mathrm{dSph}$ galaxies in general, or for Carina in particular. Venn et al. (2012) report measurements of one $\mathrm{O}$ line, and also $\mathrm{Na}$ I determinations for a few stars, but no anti-correlation clearly stems from the data. On the other hand, more metal-poor stars, with $[\mathrm{Fe} / \mathrm{H}]<-2$ dex, seem to have lower Na content than more metal-rich ones (Venn et al. 2012, their Figure 12), in agreement with what was found in GCs. Overall, $\mathrm{C}$ and $\mathrm{N}$ abundances are yet not available. These can be crucial elements, as the overall $\mathrm{C}+\mathrm{N}+\mathrm{O}$ abundance significantly influences the luminosity of the SGB and hence affects the age determinations of the different populations (Cassisi et al. 2008; Ventura et al. 2009; Marino et al. 2012). Measurements of the overall $\mathrm{C}+\mathrm{N}+\mathrm{O}$ content of intermediateage and old stars in Carina are mandatory to properly estimate the age of its stellar populations and thus to properly calibrate the chemical enrichment history.

\subsection{Comparison with Previous Results}

Following the referee's suggestion, we show in Figure 10 a comparison with literature values, in particular for the metallicity (top panel) and the age (bottom). The top panel shows the $V-c_{\mathrm{U}, \mathrm{B}, \mathrm{I}}$ diagram, with the two groups of stars selected in Section 2.2 highlighted in green and magenta. The starred symbols show the 35 stars by Lemasle et al. (2012), while the squares show the 44 stars by Fabrizio et al. (2012; 11 objects in common) color-coded as labeled. The above data indicate that the most metal-poor stars attain the lowest $c_{\mathrm{U}, \mathrm{B}, \mathrm{I}}$ values, and fall on the RGB sequence identified by the green circles. On the other hand, stars more metal-rich than $[\mathrm{Fe} / \mathrm{H}] \sim-2$ attain larger $c_{\mathrm{U}, \mathrm{B}, \mathrm{I}}$ values, and all but three fall on the other sequence identified by the magenta circles.

The bottom panel of Figure 10 shows once again the two groups of stars selected in Section 2.2 in green and in magenta together with the 35 stars by Lemasle et al. (2012). The spectroscopic sample by Fabrizio et al. (2012) was not plotted in this panel, because the authors did not provide an individual age estimate. Interestingly, we found that the most metal-poor stars also have the largest age (saturated at $15 \mathrm{Gyr}$ ). This implies
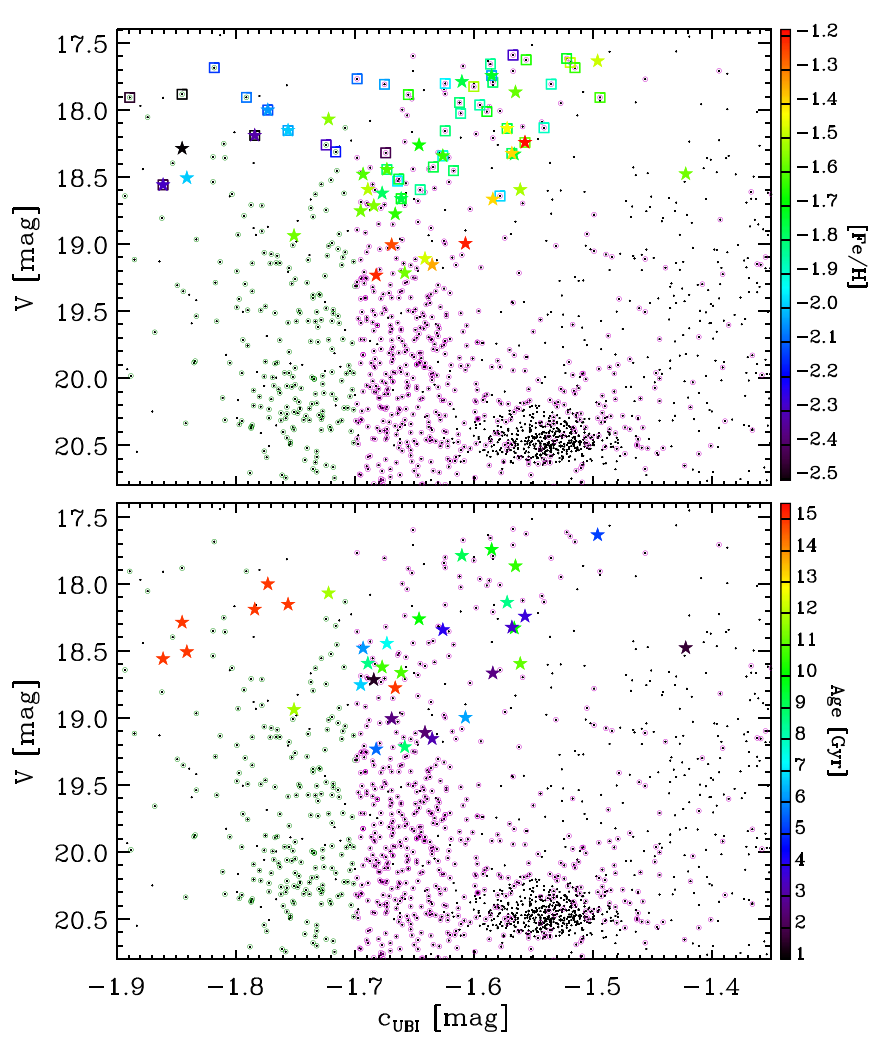

Figure 10. $V-c_{\mathrm{U}, \mathrm{B}, \mathrm{I}}$ diagram for bright Carina stars. The green and magenta circles show the separation at $c_{\mathrm{U}, \mathrm{B}, \mathrm{I}}=-1.70 \mathrm{mag}$ performed in Figure 4 . The starred symbols highlight the 35 stars from Lemasle et al. (2012). The color code used for these symbols, reproduced on the right of both panels, displays the iron content (top) and age (bottom) determined by Lemasle et al. (2012).

(A color version of this figure is available in the online journal.)

that all but one of these objects are located in the lowest $c_{\mathrm{U}, \mathrm{B}, \mathrm{I}}$ sequence, which we identify with the RGB of the old population. Similarly, all of the intermediate-age stars $(t<8 \mathrm{Gyr})$ cluster in the region of the higher $c_{\mathrm{U}, \mathrm{B}, \mathrm{I}}$ stars. On the other hand, starred symbols plotted in green with estimated ages between $\sim 8$ and $\sim 11$ Gyr attain a $c_{\mathrm{U}, \mathrm{B}, \mathrm{I}}$ index ranging from $\sim-1.75$ to $\sim-1.42$ mag. In this context it is worth mentioning that the current comparison does not allow us to reach a firm conclusion concerning the objects with ages ranging from 8 to $11 \mathrm{Gyr}$. The reasons are manifold. The uncertainty associated with the individual ages of $\mathrm{RG}$ stars is at least of the order of $2 \mathrm{Gyr}$ (Lemasle et al. 2012). The uncertainty on the individual iron abundance is at least of the order of 0.1 dex (Fabrizio et al. 2012). The sample is limited and these objects are located across the boundary adopted to split the old and the intermediate-age population.

Figure 11 shows the same $[\mathrm{Fe} / \mathrm{H}]$ abundance (top) and age (bottom) as a function of the $\Delta c_{\mathrm{U}, \mathrm{B}, \mathrm{I}}$ index, with the same color code adopted for metallicity in the top panel of Figure 10. The above figures suggest a segregation, in the sense that stars with more negative $\Delta c_{\mathrm{U}, \mathrm{B}, \mathrm{I}}$ are systematically older and more metalpoor than those with a higher index. This further strengthens the use of $c_{\mathrm{U}, \mathrm{B}, \mathrm{I}}$ and $\Delta c_{\mathrm{U}, \mathrm{B}, \mathrm{I}}$ to separate stars from different populations. However, this result should be cautiously treated, since other parameters together with age and iron content are necessary to fully characterize the properties of the Carina stars (see Figure 8 and Section 3.2). This means that the comparison between isochrones and the CMD along the RGB might be affected by possible systematic effects. The anonymous referee noted that the correlation between age and metallicity is a thorny 


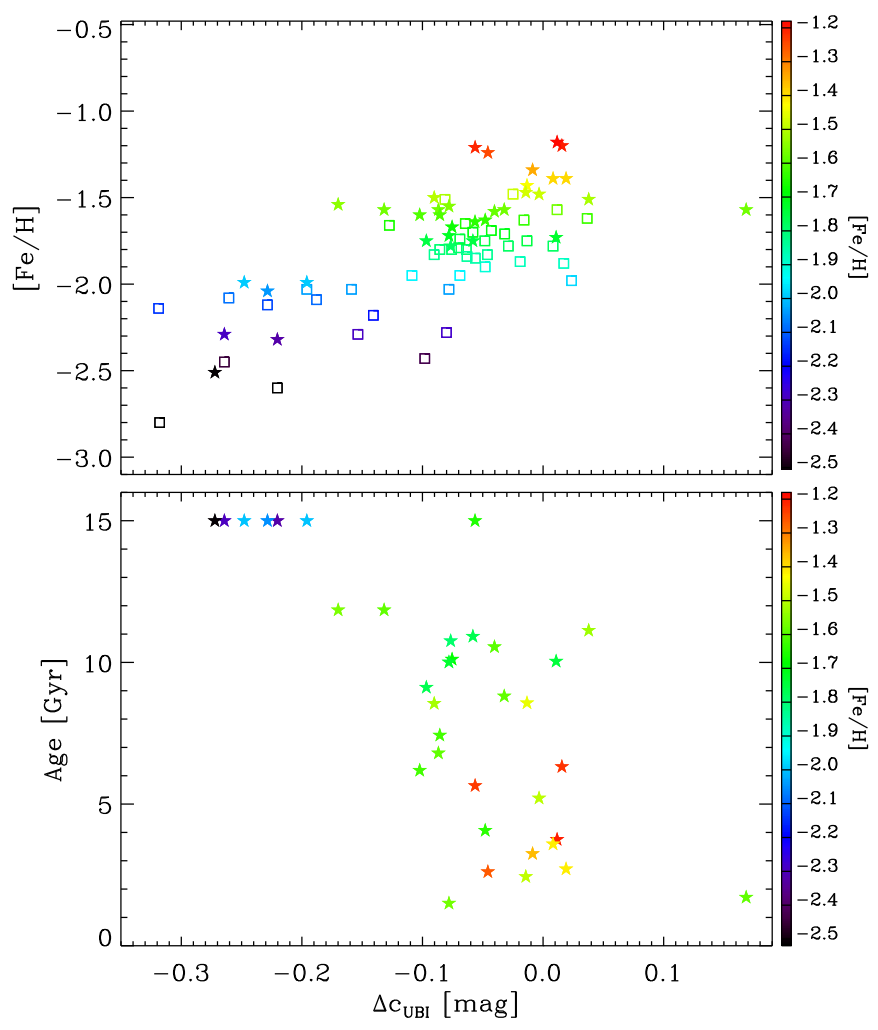

Figure 11. Iron abundance (top panel) and age (bottom) from Lemasle et al. (2012) as a function of the $\Delta c_{\mathrm{U}, \mathrm{B}, \mathrm{I}}$ index. The color code used in both panels accounts for the metallicity of the 35 stars, and is the same as that used in the top panel of Figure 10. The error bars account for the photometric error of our photometry and the uncertainties provided by Lemasle et al. (2012) on their age and metallicity determinations.

(A color version of this figure is available in the online journal.)

problem for the Carina $\mathrm{dSph}$, since the two main episodes of star formation show a well-defined separation of the order of 3-4 Gyr. According to the recent literature, the age distribution of the two main stellar populations in Carina cover a broad range in age (Mighell 1990, 1997; Smecker-Hane et al. 1994, 1996; Hurley-Keller et al. 1998; Hernandez et al. 2000; Dolphin 2002; Rizzi et al. 2003; Monelli et al. 2003; Bono et al. 2010; Stetson et al. 2011; Lemasle et al. 2012). The difference is mainly due to the adopted stellar isochrones and to the diagnostic adopted to constrain the age (MSTO versus red giants). Unfortunately, we still lack firm theoretical and empirical constraints to assess whether the Carina star formation episodes were affected by the interaction with the Galaxy (Monelli et al. 2003; Pasetto et al. 2011; Fabrizio et al. 2011; Lemasle et al. 2012). However, the above uncertainties do not affect our conclusions, since we are only using relative ages to separate the old and the intermediateage stellar populations.

The quoted problems do not apply to other satellite dSphs, such as Sculptor, showing a well-defined age-metallicity relation (de Boer et al. 2012; Starkenburg et al. 2013). This stellar system appears as a fundamental benchmark to further constrain the use of the $c_{\mathrm{U}, \mathrm{B}, \mathrm{I}}$ index to identify distinct stellar populations.

\section{CONCLUSIONS}

In this paper, we have investigated the stellar populations in Carina, adopting an observational approach that has been successfully used to study multiple populations in GCs. The main results can be summarized as follows.
1. The use of the $c_{\mathrm{U}, \mathrm{B}, \mathrm{I}}$ pseudo-color allows for discrimination between old and intermediate-age populations. The new purely photometric diagnostic allows us to break the severe age-metallicity degeneracy along a significant portion of the RGB of this stellar system. In particular, we are able to clearly follow the evolutionary sequence of the old and intermediate-age populations for at least three magnitudes along the RGB. This approach is fully supported by the analysis sample of bona fide Carina stars selected according to their radial velocity.

2. By comparing with published spectroscopy, we found a significant correlation between the $c_{\mathrm{U}, \mathrm{B}, \mathrm{I}}$ index of RGB stars and their iron content. This conclusion is supported by three independent samples of spectroscopic measurements. We derive that the mean metallicity of the old population is on average 0.5 dex lower than the intermediate-age one.

3. A comparison with theoretical models demonstrates that current isochrones calculated for a standard heavy-element mixture cannot reproduce the observed distribution of stars in the $\left(V, c_{\mathrm{U}, \mathrm{B}, \mathrm{I}}\right)$ plane.

4. The appearance of Carina RGBs in different CMDs significantly differs from what is observed for multiple populations within GCs.

The comparison between theoretical models and observations indicates that the RGB bimodality disclosed by $c_{\mathrm{U}, \mathrm{B}, \mathrm{I}}$ cannot be explained on the basis of a difference in age and/or in Fe abundance only. In dealing with mono-metallic GCs, the split in the different subpopulations is currently explained as the combination of light-element (mainly C, N, O) and helium variations (e.g., Marino et al. 2008; Yong \& Grundahl 2008; Sbordone et al. 2011; Milone et al. 2012a). Very likely, a possible difference in $\mathrm{C}, \mathrm{N}, \mathrm{O}$ - and probably helium-between the old and young population, combined with metallicity and age effects, could also be responsible for the observed RGB bimodality in Carina. However, in the case of Carina, there are no measurements for most of these elements. In addition, while in GCs the C, N, O abundances are connected with wellknown correlations and anti-correlations, the pattern of $\mathrm{C}, \mathrm{N}, \mathrm{O}$ variations in this dwarf is largely unexplored (e.g., Venn et al. 2012) and the empirical scenario is still limited by both accuracy and statistics.

Detailed knowledge of the chemical pattern in dwarf galaxies, especially in comparison with GCs, is very important to constrain the chemical evolution in the Milky Way environment. The techniques shown here could be very promising for breaking the age-metallicity degeneracy in the CMDs of stellar systems with a complex star formation history, and should be tested in different systems. In particular, comparing Carina with other galaxies with different star formation histories, such as the purely old ones (Draco, Ursa Minor, Sculptor) and those with extended star formation (Fornax, Leo I), would be particularly valuable.

Support for this work has been provided by the Education and Science Ministry of Spain (grant AYA2010-16717). A.P.M. acknowledges financial support from the Australian Research Council through Discovery Project grant DP120100475. S.C. is grateful for financial support from PRIN-INAF 2011 "Multiple Populations in Globular Clusters: their role in the Galaxy assembly" (PI: E. Carretta), and from PRIN MIUR 2010-2011, project "The Chemical and Dynamical Evolution of the Milky Way and Local Group Galaxies," prot. 2010LY5N2T (PI: F. Matteucci). M.F. acknowledges financial support from the PO FSE Abruzzo 
2007-2013 through the grant "Spectro-photometric characterization of stellar populations in Local Group dwarf galaxies," prot.89/2014/OACTe/D (PI: S. Cassisi).

\section{REFERENCES}

Aparicio, A., Carrera, R., \& Martínez-Delgado, D. 2001, AJ, 122, 2524

Battaglia, G., Irwin, M., Tolstoy, E., de Boer, T., \& Mateo, M. 2012, ApJL, 761, L31

Bedin, L. R., Piotto, G., Anderson, J., et al. 2004, ApJL, 605, L125

Bekki, K., Campbell, S. W., Lattanzio, J. C., \& Norris, J. E. 2007, MNRAS, 377, 335

Bellazzini, M., Ferraro, F. R., \& Pancino, E. 2001, MNRAS, 327, L15

Bono, G., Stetson, P. B., Walker, A. R., et al. 2010, PASP, 122, 651

Cannon, R. D., Croke, B. F. W., Bell, R. A., Hesser, J. E., \& Stathakis, R. A. 1998, MNRAS, 298, 601

Carrera, R., Aparicio, A., Martínez-Delgado, D., \& Alonso-García, J. 2002, AJ, 123,3199

Cassisi, S., Mucciarelli, A., Pietrinferni, A., Salaris, M., \& Ferguson, J. 2013a, A\&A, 554, A19

Cassisi, S., Salaris, M., \& Pietrinferni, A. 2013b, MmSAI, 84, 91

Cassisi, S., Salaris, M., Pietrinferni, A., et al. 2008, ApJ, 672, L115

Cole, A. A., Skillman, E. D., Tolstoy, E., et al. 2007, ApJL, 659, L17

Coppola, G., Stetson, P. B., Marconi, M., et al. 2013, ApJ, 775, 6

Dall'Ora, M., Kinemuchi, K., Ripepi, V., et al. 2012, ApJ, 752, 42

de Boer, T. J. L., Tolstoy, E., Hill, V., et al. 2012, A\&A, 539, A103

D’Ercole, A., Vesperini, E., D’Antona, F., McMillan, S. L. W., \& Recchi, S. 2008, MNRAS, 391, 825

Dolphin, A. E. 2002, MNRAS, 332, 91

Dolphin, A. E., Walker, A. R., Hodge, P. W., et al. 2001, ApJ, 562, 303

Fabrizio, M., Merle, T., Thévenin, F., et al. 2012, PASP, 124, 519

Fabrizio, M., Nonino, M., Bono, G., et al. 2011, PASP, 123, 384

Gallagher, J. S., Tolstoy, E., Dohm-Palmer, R. C., et al. 1998, AJ, 115,1869

Gallart, C., Aparicio, A., \& Vilchez, J. M. 1996, AJ, 112, 1928

Grundahl, F., Catelan, M., Landsman, W. B., Stetson, P. B., \& Andersen, M. I. 1999, ApJ, 524, 242

Held, E. V., Saviane, I., \& Momany, Y. 1999, A\&A, 345, 747

Helmi, A., Irwin, M. J., Tolstoy, E., et al. 2006, ApJL, 651, L121

Hernandez, X., Gilmore, G., \& Valls-Gabaud, D. 2000, MNRAS, 317, 831

Hidalgo, S. L., Aparicio, A., Skillman, E., et al. 2011, ApJ, 730, 14

Hurley-Keller, D., Mateo, M., \& Nemec, J. 1998, AJ, 115, 1840

Koch, A., Grebel, E. K., Wyse, R. F. G., et al. 2006, AJ, 131, 895
Kraft, R. P., Sneden, C., Langer, G. E., \& Prosser, C. F. 1992, AJ, 104, 645

Lee, J.-W., Kang, Y.-W., Lee, J., \& Lee, Y.-W. 2009, Natur, 462, 480

Lemasle, B., Hill, V., Tolstoy, E., et al. 2012, A\&A, 538, A100

Marino, A. F., Milone, A. P., Sneden, C., et al. 2012, A\&A, 541, A15

Marino, A. F., Villanova, S., Piotto, G., et al. 2008, A\&A, 490, 625

Martin, N. F., McConnachie, A. W., Irwin, M., et al. 2009, ApJ, 705, 758

McConnachie, A. W., \& Irwin, M. J. 2006, MNRAS, 365, 1263

Mighell, K. J. 1990, A\&AS, 82, 1

Mighell, K. J. 1997, AJ, 114, 1458

Milone, A. P., Marino, A. F., Cassisi, S., et al. 2012a, ApJL, 754, L34

Milone, A. P., Marino, A. F., Piotto, G., et al. 2012b, ApJ, 745, 27

Milone, A. P., Piotto, G., Bedin, L. R., et al. 2012c, A\&A, 537, A77

Milone, A. P., Bedin, L. R., Piotto, G., et al. 2008, ApJ, 673, 241

Momany, Y., Held, E. V., Saviane, I., et al. 2005, A\&A, 439, 111

Monelli, M., Cassisi, S., Bernard, E. J., et al. 2010a, ApJ, 718, 707

Monelli, M., Hidalgo, S. L., Stetson, P. B., et al. 2010b, ApJ, 720, 1225

Monelli, M., Milone, A. P., Stetson, P. B., et al. 2013, MNRAS, 431, 2126

Monelli, M., Pulone, L., Corsi, C. E., et al. 2003, AJ, 126, 218

Okamoto, S., Arimoto, N., Yamada, Y., \& Onodera, M. 2012, ApJ, 744, 96

Pasetto, S., Grebel, E. K., Berczik, P., Chiosi, C., \& Spurzem, R. 2011, A\&A, 525, A99

Pietrinferni, A., Cassisi, S., Salaris, M., \& Castelli, F. 2004, ApJ, 612, 168

Piotto, G., Milone, A. P., Anderson, J., et al. 2012, ApJ, 760, 39

Rizzi, L., Held, E. V., Bertelli, G., \& Saviane, I. 2003, ApJL, 589, L85

Rizzi, L., Held, E. V., Saviane, I., Tully, R. B., \& Gullieuszik, M. 2007, MNRAS, 380, 1255

Sanna, N., Bono, G., Stetson, P. B., et al. 2010, ApJL, 722, L244

Sbordone, L., Salaris, M., Weiss, A., \& Cassisi, S. 2011, A\&A, 534, A9

Shetrone, M., Venn, K. A., Tolstoy, E., et al. 2003, AJ, 125, 684

Shetrone, M. D., Côté, P., \& Sargent, W. L. W. 2001, ApJ, 548, 592

Skillman, E. D., Tolstoy, E., Cole, A. A., et al. 2003, ApJ, 596, 253

Smecker-Hane, T. A., Stetson, P. B., Hesser, J. E., \& Lehnert, M. D. 1994, AJ, 108, 507

Smecker-Hane, T. A., Stetson, P. B., Hesser, J. E., \& Vandenberg, D. A. 1996, in ASP Conf. Ser. 98, From Stars to Galaxies: the Impact of Stellar Physics on Galaxy Evolution, ed. C. Leitherer, U. Fritze-von-Alvensleben, \& J. Huchra (San Francisco, CA: ASP), 328

Starkenburg, E., Hill, V., Tolstoy, E., et al. 2013, A\&A, 549, A88

Stetson, P. B., Monelli, M., Fabrizio, M., et al. 2011, Msngr, 144, 32

Tolstoy, E., Venn, K. A., Shetrone, M., et al. 2003, AJ, 125, 707

Venn, K. A., Shetrone, M. D., Irwin, M. J., et al. 2012, ApJ, 751, 102

Ventura, P., Caloi, V., D'Antona, F., et al. 2009, MNRAS, 399, 934

Walker, M. G., Mateo, M., \& Olszewski, E. W. 2009, AJ, 137, 3100

Yong, D., \& Grundahl, F. 2008, ApJL, 672, L29 